\title{
Factors That Influence the Use of Electronic Diaries in Health Care: Scoping Review
}

Naomi E M Daniëls ${ }^{1,2}$, MSc; Laura M J Hochstenbach ${ }^{3}, \mathrm{PhD}$; Catherine van Zelst ${ }^{1}, \mathrm{PhD}$; Marloes A van Bokhoven ${ }^{2}$, MD, PhD; Philippe A E G Delespaul ${ }^{1,4}, \mathrm{PhD}$; Anna J H M Beurskens ${ }^{2}$, PhD

\footnotetext{
${ }^{1}$ Department of Psychiatry and Neuropsychology, Faculty of Health, Medicine and Life Sciences, Maastricht University, Maastricht, Netherlands

${ }^{2}$ Department of Family Medicine, Faculty of Health, Medicine and Life Sciences, Maastricht University, Maastricht, Netherlands

${ }^{3}$ Research Centre for Remote Health Care, Faculty of Health Care, Zuyd University of Applied Sciences, Heerlen, Netherlands

${ }^{4}$ Mondriaan Mental Health Trust, Heerlen/Maastricht, Netherlands
}

\section{Corresponding Author:}

Naomi E M Daniëls, MSc

Department of Psychiatry and Neuropsychology

Faculty of Health, Medicine and Life Sciences

Maastricht University

Vijverdalseweg 1

Maastricht, $6226 \mathrm{NB}$

Netherlands

Phone: 31433883820

Email: naomi.daniels@maastrichtuniversity.nl

\section{Abstract}

Background: A large number of people suffer from psychosocial or physical problems. Adequate strategies to alleviate needs are scarce or lacking. Symptom variation can offer insights into personal profiles of coping and resilience (detailed functional analyses). Hence, diaries are used to report mood and behavior occurring in daily life. To reduce inaccuracies, biases, and noncompliance with paper diaries, a shift to electronic diaries has occurred. Although these diaries are increasingly used in health care, information is lacking about what determines their use.

Objective: The aim of this study was to map the existing empirical knowledge and gaps concerning factors that influence the use of electronic diaries, defined as repeated recording of psychosocial or physical data lasting at least one week using a smartphone or a computer, in health care.

Methods: A scoping review of the literature published between January 2000 and December 2018 was conducted using queries in PubMed and PsycInfo databases. English or Dutch publications based on empirical data about factors that influence the use of electronic diaries for psychosocial or physical purposes in health care were included. Both databases were screened, and findings were summarized using a directed content analysis organized by the Consolidated Framework for Implementation Research (CFIR).

Results: Out of 3170 articles, 22 studies were selected for qualitative synthesis. Eleven themes were determined in the CFIR categories of intervention, user characteristics, and process. No information was found for the CFIR categories inner (eg, organizational resources, innovation climate) and outer (eg, external policies and incentives, pressure from competitors) settings. Reminders, attractive designs, tailored and clear data visualizations (intervention), smartphone experience, and intrinsic motivation to change behavior (user characteristics) could influence the use of electronic diaries. During the implementation process, attention should be paid to both theoretical and practical training.

Conclusions: Design aspects, user characteristics, and training and instructions determine the use of electronic diaries in health care. It is remarkable that there were no empirical data about factors related to embedding electronic diaries in daily clinical practice. More research is needed to better understand influencing factors for optimal electronic diary use.

(JMIR Mhealth Uhealth 2021;9(6):e19536) doi: $\underline{10.2196 / 19536}$

\section{KEYWORDS}

compliance; delivery of health care; diary; ecological momentary assessment; intention; motivation; scoping review 


\section{Introduction}

Health care professionals are insufficiently aware of symptom variability and contextual fluctuations; therefore, their interventions are based on incomplete information [1-5]. Patients are asked to recall their mood, thoughts, behavior, and experiences over the past weeks or even months. Recalling information from memory, though, is known to be incomplete and inaccurate $[6,7]$. To minimize inaccuracies and biases, prospective diaries are used to collect patients' mood, thoughts, behavior, and experiences in the relevant context close to the time of occurrence [8]. Because these health-related strategies often require management of vulnerabilities, long-term patient engagement is important. However, patients experience that it is difficult to be engaged in the use of diaries for long periods of time. Compliance is often poor, and adequate reports on contextual variation are lacking [8]. Paper diaries are remarkably completed in the parking lot before meeting the clinician [9]. In one-third of the days, paper diaries contain entries while the $\log$ booklets were not opened $[8,10]$.

To overcome noncompliance with paper diaries, researchers and clinicians have shifted from paper to electronic diaries. Both paper and electronic diaries can be used in research to observe individuals in their context, gather data about sensitive topics, or to actively engage individuals in monitoring and reflecting on behaviors, their underlying mechanisms, and processes. Furthermore, these diaries can be implemented in intervention studies, clinical trials, and routine care [11,12]. Electronic diaries are, however, more reliable and logistically easier to implement $[13,14]$. They allow individuals to monitor in daily life with little retrospection and reduced obtrusiveness. Electronic diaries are signal-contingent and often record response-time information, which improves reliability [15-18]. Nonetheless, electronic diaries also have disadvantages. Development and maintenance are costly [12]. Technical problems occur, and not all patients are acquainted with smartphones and require instructions and coaching [15]. Furthermore, research on compliance is ambiguous. For instance, the percentage of completed diary entries with electronic diaries ranges from less than $50 \%$ to $99 \%$ [18-20]. High participant motivation is related to accurate data collection and less faked compliance [13].

Previous research states that various factors are related to the use of electronic diaries, such as the design (ie, ease of use, entertainment value), the social context (ie, satisfaction and connection with others), and the user's characteristics (ie, education and self-efficacy) [21-23]. However, no complete overview is available concerning empirical data about the factors related to the use of these tools. Therefore, the main aim of this paper was to map the existing empirical knowledge about the factors that influence the use of electronic diaries in health care. Electronic diaries in health care were defined as repeated individual psychosocial or physical data collection using measurement tools on a smartphone (applications) or on a computer (website), including among others, experience sampling, ambulatory assessment, and ecological momentary assessment. In addition, use was defined as the repeated recording of information in electronic diaries by patients or healthy individuals for at least one week, including adherence, compliance, and engagement. The cut-off point was determined based on the expected recall bias and necessary data for comprehensive functional diagnostics.

\section{Methods}

In order to map existing knowledge concerning the topic of interest and to identify any gaps, this scoping review was based on the methodological framework proposed by Arksey and O'Malley [24]. This framework includes 5 specific steps: identify the research question, identify relevant studies, select relevant studies, chart the data, and summarize and report the results. The selection of relevant studies was not based on methodological quality, but on relevance.

\section{Identify the Research Question}

The research question of this scoping review was based on prior research and the expertise of the research team. It is summarized as: "What is the current empirical knowledge regarding factors that influence the use of electronic diaries in health care?"

\section{Identify and Select Relevant Studies}

A structured literature search was conducted using the PubMed and PsycInfo databases to search for articles published between 2000 and 2018. The search was limited to human adults and articles published in Dutch or English. Both free-text search terms and MESH headings were used. The search strategy included 2 different concepts: "continued use" and "electronic diaries." The search string used is depicted in Textbox 1. In addition to the database search, reference lists of relevant studies were screened manually for further relevant papers. This is a valuable step (snowball method) to identify articles that have been missed in the database search because electronic databases may be incomplete and they can vary in coverage, indexing, and depth of information [24]. Moreover, 2 experts in the field were contacted to identify key authors or key publications on the topic of interest. 
Textbox 1. Search string.

\author{
1. Use: "compliance (MeSH) OR intention (MeSH) OR motivation (MeSH) OR 'continued usage' OR use OR continuance OR adherence OR \\ engagement"; \\ AND \\ 2. Electronic diaries: "momentary (MeSH: ecological momentary assessment) OR 'real time data' (MeSH) OR e-diaries OR electronic diar* OR \\ structured diar* OR computer diar* OR 'experience sampling' OR ambulatory assessment OR electronic assessment* OR electronic interview* OR \\ self-monitoring" \\ Limits: \\ - Publication date: $2000-2018$ \\ - Humans: adult \\ - $\quad$ Language: English, Dutch
}

Two researchers (NEMD, LMJH) reviewed the retrieved studies using a 3-step screening process: titles, abstracts, and full articles. The screening process of a scoping review is not linear but rather iterative, which required the researchers to engage with each step in a reflexive way and repeat steps to ensure that the literature was covered in an extensive way. If the relevance of a study was unclear from the title, the abstract was ordered, and if the relevance of a study was unclear from the abstract, the full article was ordered. As a check on the 3-step screening process, we read the full texts of a random sample of 50 titles and 50 abstracts. In only 4 articles, we found information in the results or the discussion related to our scope. Relevant studies with the following criteria were included: (1) using electronic diaries for psychosocial or physical data, (2) describing factors that influence the use of electronic diaries, and (3) a focus on health care. No methodological criteria were applied, and articles based on empirical data were included. Studies were excluded when the definitions of electronic diaries or use in the article did not match with the ones used in this manuscript (ie, the data collection method: single moment data collection or passive self-monitoring using sensors, activity trackers, or biomarkers). Studies that used a combination of active and passive monitoring were not excluded. Moreover, studies were excluded when the article did not include factors that influence the use of electronic diaries as the outcome (ie, the study aim: experiences with disease management, epidemiology, health technology assessment, prediction models, outcome and effect studies, and the study design [reviews, secondary analysis, protocols]). Studies in which disease management were based on or complemented with self-reporting and studies about technology acceptance were not excluded. Furthermore, we excluded studies with a target population other than adults. For children and adolescents, we expect that different factors influence the use of electronic diaries specifically and interventions in general as parents, for instance, need to give their permission. At each step, the articles were categorized as relevant, irrelevant, and dubious according to the aforementioned exclusion criteria. Differences were discussed until consensus was reached. When no consensus was reached or questions remained, a third researcher $(\mathrm{CvZ})$ was consulted.

\section{Chart the Data}

The data were charted using Excel spreadsheets and included study details (author, title, database, journal, year of publication, study location [published and conducted], study population and sample size, study aims, design, and setting), intervention characteristics (aim, content, and duration of the electronic diary), and key findings (factors that influence the use). These factors were organized according to the Consolidated Framework for Implementation Research (CFIR) [25]. This framework consists of 5 categories (ie, intervention characteristics, outer setting, inner setting, individual characteristics, and process) related to sustainable implementation. The intervention characteristics category includes, among others, the complexity of the electronic diary or the ability to test the electronic diary on a small scale. The outer setting category is comprised of the economic, political, and social context of the organization. The inner setting category includes, among others, the internal architecture of the organization and the innovation climate. The individual characteristics category is comprised of, among others, the individual's knowledge, beliefs, and self-efficacy regarding the intervention or the implementation process. The process category includes activities (planning, engaging, executing, reflecting, and evaluating) related to the implementation process.

\section{Summarize and Report the Results}

Content analysis was done independently by 2 reviewers (NEMD, LMJH) based on the 5 categories of the CFIR [25]: (1) intervention, (2) outer setting, (3) inner setting, (4) individual characteristics, and (5) process. Directed content analysis, using inductive reasoning, was used to validate or conceptually extend the framework [26]. The themes were based on our previous work [27] and emerged from the data. After coding, the researchers compared their codes until consensus was reached. They identified key themes into which the results could be divided.

\section{Results}

The database search resulted in 3650 hits (Figure 1). After removing duplicates and reviewing 3170 titles, 273 abstracts were screened, of which 50 full texts were evaluated. In total, 20 articles were included based on the predefined eligibility criteria. Two articles were included from the additional hand search, which resulted in 22 articles in total for qualitative synthesis. The publication patterns are summarized in Multimedia Appendix 1. 
Figure 1. Scoping review flow diagram.
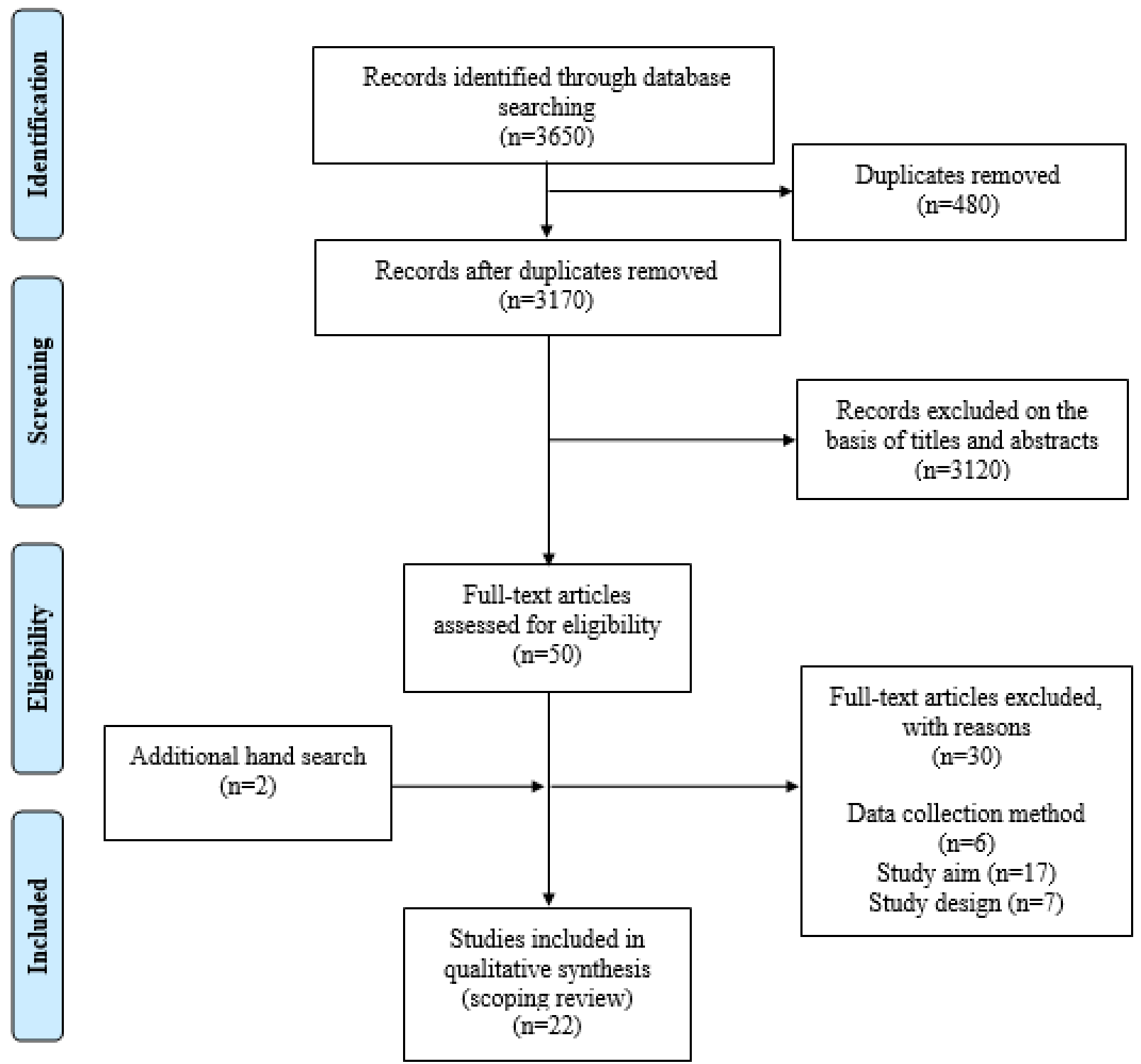

\section{Electronic Diary and Study Characteristics}

More detailed information about the content of the 22 selected studies with empirical data on factors that influence the use of electronic diaries in health care can be found in Table 1. Electronic diaries were used either to monitor one's own behavior in order to get insight into underlying patterns or mechanisms (monitoring: 12/22, 55\%) or to actively achieve change (intervention: 10/22, 45\%). They mainly focused on measuring lifestyle behaviors (14/22, 64\%) and constructs such as pain or mood. Participants completed these electronic diaries via palmtop $(3 / 22,14 \%)$, smartphone $(14 / 22,64 \%)$, or (tablet) computer $(5 / 22,22 \%)$. The assessment frequency ranged from 12 times a day, an example of the experience sampling method or ecological momentary assessment (EMA), to weekly, and the duration of the data collection varied from 2 weeks to 2.5 years. 
Table 1. Electronic diary (e-diary) and study characteristics.

\begin{tabular}{|c|c|c|c|c|c|c|}
\hline \multirow{2}{*}{$\begin{array}{l}\text { First author, year, } \\
\text { country }\end{array}$} & \multicolumn{3}{|l|}{ e-Diary characteristics } & \multicolumn{3}{|l|}{ Study characteristics } \\
\hline & $\begin{array}{l}\text { Purpose of use }{ }^{a} \text { (de- } \\
\text { vice) }\end{array}$ & $\begin{array}{l}\text { Constructs mea- } \\
\text { sured }\end{array}$ & $\begin{array}{l}\text { Frequency of use and } \\
\text { duration }\end{array}$ & Study aims & $\begin{array}{l}\text { Design and data } \\
\text { collection }\end{array}$ & $\begin{array}{l}\text { Sample: target } \\
\text { population, num- } \\
\text { ber of partici- } \\
\text { pants, sex, age } \\
\text { (years) }\end{array}$ \\
\hline $\begin{array}{l}\text { Litcher-Kelly, } \\
2007 \text { [29], US }\end{array}$ & $\begin{array}{l}\text { Monitoring: self-moni- } \\
\text { toring diaries (palmtop) }\end{array}$ & $\begin{array}{l}\text { Mood, stress, } \\
\text { pain, medication } \\
\text { use }\end{array}$ & $\begin{array}{l}12 \text { times a day for } 3 \\
\text { weeks }\end{array}$ & $\begin{array}{l}\text { Feasibility of an } \\
\text { electronic diary }\end{array}$ & $\begin{array}{l}\text { Quantitative: in- } \\
\text { tervention study } \\
\text { with continuous } \\
\text { log data }\end{array}$ & $\begin{array}{l}\text { Patients with in- } \\
\text { flammatory bow- } \\
\text { el disease }(n=16) \text {, } \\
25 \% \text { male }(n=4), \\
\text { mean age } 46.0 \\
(\text { SD } 13.6)\end{array}$ \\
\hline $\begin{array}{l}\text { Welch, } 2007 \\
{[30], \text { US }}\end{array}$ & $\begin{array}{l}\text { Monitoring: self-moni- } \\
\text { toring diaries (palmtop) }\end{array}$ & $\begin{array}{l}\text { Food and fluid } \\
\text { intake }\end{array}$ & $\begin{array}{l}3 \text { times a day for } 12 \\
\text { weeks }\end{array}$ & $\begin{array}{l}\text { Feasibility of elec- } \\
\text { tronic self-monitor- } \\
\text { ing diaries }\end{array}$ & $\begin{array}{l}\text { Quantitative: pi- } \\
\text { lot study with } \\
\text { surveys }\end{array}$ & $\begin{array}{l}\text { Patients on } \\
\text { hemodialysis } \\
(\mathrm{n}=3), 67 \% \text { male } \\
(\mathrm{n}=2), \text { mean age } \\
54\end{array}$ \\
\hline $\begin{array}{l}\text { Stevens, } 2008 \\
\text { [31], US }\end{array}$ & $\begin{array}{l}\text { Intervention: } \mathrm{IT}^{\mathrm{f}} \text { weight } \\
\text { loss program (comput- } \\
\text { er) }\end{array}$ & $\begin{array}{l}\text { Weight, food } \\
\text { records, exercise } \\
\text { minutes }\end{array}$ & $\begin{array}{l}\text { Weekly for a } 2.5 \text {-year } \\
\text { follow-up }\end{array}$ & $\begin{array}{l}\text { First year utiliza- } \\
\text { tion and develop- } \\
\text { ment process of an } \\
\text { IT weight loss pro- } \\
\text { gram }\end{array}$ & $\begin{array}{l}\text { Quantitative: } \\
\text { RCT with } 3 \\
\text { groups (no-fur- } \\
\text { ther treatment, } \\
\text { control condition, } \\
\text { or active mainte- } \\
\text { nance weight loss } \\
\text { intervention) }\end{array}$ & $\begin{array}{l}\text { Adults with a } \\
\text { BMI of } 25-45 \\
\mathrm{~kg} / \mathrm{m}^{2} \text { who were } \\
\text { taking medication } \\
\text { for hypertension } \\
\text { or hyperlipidemia } \\
(\mathrm{n}=348), 37 \% \\
\text { male }(\mathrm{n}=128) \text {, } \\
\text { mean age } 56\end{array}$ \\
\hline $\begin{array}{l}\text { Webber, } 2010 \\
\text { [32], US }\end{array}$ & $\begin{array}{l}\text { Monitoring: internet } \\
\text { behavioral weight loss } \\
\text { program (computer) }\end{array}$ & $\begin{array}{l}\text { Daily caloric in- } \\
\text { take, daily exer- } \\
\text { cise, weight }\end{array}$ & $\begin{array}{l}\text { At least weekly for } 16 \\
\text { weeks }\end{array}$ & $\begin{array}{l}\text { Motivation and ad- } \\
\text { herence to self- } \\
\text { monitoring and } \\
\text { weight loss }\end{array}$ & $\begin{array}{l}\text { Quantitative: sec- } \\
\text { ondary analysis } \\
\text { of existing RCT } \\
\text { data (did or did } \\
\text { not achieve } 5 \% \\
\text { weight loss) }\end{array}$ & $\begin{array}{l}\text { Adult women } \\
\text { with a BMI of } \\
25-40 \mathrm{~kg} / \mathrm{m}^{2} \\
(\mathrm{n}=66), \text { mean age } \\
50.1(\text { SD } 9.9)\end{array}$ \\
\hline $\begin{array}{l}\text { Ahtinen, } 2013 \\
\text { [33], Finland }\end{array}$ & $\begin{array}{l}\text { Intervention: Oiva, a } \\
\text { mobile mental wellness } \\
\text { training application } \\
\text { (smartphone) }\end{array}$ & $\begin{array}{l}\text { Reflections and } \\
\text { notes on exercis- } \\
\text { es }\end{array}$ & Daily for a month & $\begin{array}{l}\text { Use, acceptance, } \\
\text { and usefulness of } \\
\text { Oiva }\end{array}$ & $\begin{array}{l}\text { Mixed methods: } \\
\text { feasibility study } \\
\text { with surveys, app } \\
\text { log data and inter- } \\
\text { views }\end{array}$ & $\begin{array}{l}\text { Individuals inter- } \\
\text { ested in stress } \\
\text { management } \\
(n=15), 40 \% \\
\text { male }(n=6) \text {, } \\
\text { working age }\end{array}$ \\
\hline $\begin{array}{l}\text { Ben-Zeev, } 2013 \\
{[34], \text { US }}\end{array}$ & $\begin{array}{l}\text { Intervention: FOCUS, } \\
\text { a mobile illness self- } \\
\text { management system } \\
\text { (smartphone) }\end{array}$ & $\begin{array}{l}\text { Medication adher- } \\
\text { ence, mood regu- } \\
\text { lation, sleep, so- } \\
\text { cial functioning, } \\
\text { coping with per- } \\
\text { sistent auditory } \\
\text { hallucinations }\end{array}$ & Daily & $\begin{array}{l}\text { Development of } \\
\text { FOCUS }\end{array}$ & $\begin{array}{l}\text { Mixed methods: } \\
\text { usability study } \\
\text { with surveys and } \\
\text { think-aloud proce- } \\
\text { dure }\end{array}$ & $\begin{array}{l}\text { Patients with } \\
\text { schizophrenia or } \\
\text { schizoaffective } \\
\text { disorder }(n=12) \text {, } \\
67 \% \text { male }(n=8) \text {, } \\
\text { mean age } 45\end{array}$ \\
\hline
\end{tabular}




\begin{tabular}{|c|c|c|c|c|c|c|}
\hline \multirow{2}{*}{$\begin{array}{l}\text { First author, year, } \\
\text { country }\end{array}$} & \multicolumn{3}{|l|}{ e-Diary characteristics } & \multicolumn{3}{|l|}{ Study characteristics } \\
\hline & $\begin{array}{l}\text { Purpose of use }{ }^{a} \text { (de- } \\
\text { vice) }\end{array}$ & $\begin{array}{l}\text { Constructs mea- } \\
\text { sured }\end{array}$ & $\begin{array}{l}\text { Frequency of use and } \\
\text { duration }\end{array}$ & Study aims & $\begin{array}{l}\text { Design and data } \\
\text { collection }\end{array}$ & $\begin{array}{l}\text { Sample: target } \\
\text { population, num- } \\
\text { ber of partici- } \\
\text { pants, sex, age } \\
\text { (years) }\end{array}$ \\
\hline $\begin{array}{l}\text { Tatara, } 2013 \\
\text { [36], Norway }\end{array}$ & $\begin{array}{l}\text { Monitoring: Few } \\
\text { Touch, a mobile self- } \\
\text { management applica- } \\
\text { tion (smartphone) }\end{array}$ & Nutritional habits & $\begin{array}{l}1 \text { year, no app use crite- } \\
\text { ria }\end{array}$ & $\begin{array}{l}\text { Factors associated } \\
\text { with use of Few } \\
\text { Touch, a mobile } \\
\text { self-management } \\
\text { application }\end{array}$ & $\begin{array}{l}\text { Mixed methods: } \\
\text { longitudinal inter- } \\
\text { vention trial with } \\
\text { surveys, inter- } \\
\text { views, and focus } \\
\text { groups }\end{array}$ & $\begin{array}{l}\text { Individuals with } \\
\text { type } 2 \text { diabetes } \\
\text { mellitus }(\mathrm{n}=12), \\
33 \% \text { male }(\mathrm{n}=4) \text {, } \\
\text { mean age } 55.1 \\
\text { (SD 9.6) }\end{array}$ \\
\hline $\begin{array}{l}\text { Tang, } 2015 \text { [37], } \\
\text { UK }\end{array}$ & $\begin{array}{l}\text { Monitoring: publicly } \\
\text { available free applica- } \\
\text { tions MyFitness Pal, } \\
\text { Livestrong, Calorie } \\
\text { Count, SparkPeople } \\
\text { (smartphone) }\end{array}$ & Not specified & $\begin{array}{l}3 \text { weeks, no app use } \\
\text { criteria }\end{array}$ & $\begin{array}{l}\text { Understanding of } \\
\text { users' experiences } \\
\text { with weight loss or } \\
\text { weight control } \\
\text { apps }\end{array}$ & $\begin{array}{l}\text { Qualitative: } \\
\text { semistructured } \\
\text { interviews }\end{array}$ & $\begin{array}{l}\text { Young adults } \\
\text { having experi- } \\
\text { ence with or inter- } \\
\text { est in using an } \\
\text { eHealth weight } \\
\text { loss maintenance } \\
\text { app }(n=19), 54 \% \\
\text { male }(n=10) \text {, age } \\
\text { range } 19-33\end{array}$ \\
\hline $\begin{array}{l}\text { Anderson, } 2016 \\
\text { [39], Australia }\end{array}$ & $\begin{array}{l}\text { Monitoring: applica- } \\
\text { tions about chronic } \\
\text { conditions (sleep disor- } \\
\text { ders, migraine, menstru- } \\
\text { al irregularities, chronic } \\
\text { depression, arthritis and } \\
\text { Behçet's disease; } \\
\text { smartphone) }\end{array}$ & $\begin{array}{l}\text { Ranging from } \\
\text { symptom monitor- } \\
\text { ing or manage- } \\
\text { ment apps to fit- } \\
\text { ness apps }\end{array}$ & $\begin{array}{l}\text { Ranging from several } \\
\text { weeks to } 2 \text { years }\end{array}$ & $\begin{array}{l}\text { Consumers' experi- } \\
\text { ences with mobile } \\
\text { health apps }\end{array}$ & $\begin{array}{l}\text { Qualitative: indi- } \\
\text { vidual semistruc- } \\
\text { tured interviews }\end{array}$ & $\begin{array}{l}\text { Healthy individu- } \\
\text { als reporting the } \\
\text { recent use of any } \\
\text { commercially } \\
\text { available } \\
\text { health/fitness app } \\
\text { with capacity for } \\
\text { self-monitoring } \\
\text { and data input } \\
\text { (n=22), 32\% } \\
\text { male (n=7), age } \\
\text { range } 18-55\end{array}$ \\
\hline
\end{tabular}


First author, year, e-Diary characteristics country

Purpose of use ${ }^{\mathrm{a}}$ (device)
Study characteristics

Design and data collection
Constructs mea- Frequency of use and Study aims sured duration
Sample: target population, number of participants, sex, age (years)

\begin{tabular}{|c|c|c|c|c|c|c|}
\hline $\begin{array}{l}\text { Batink, } 2016 \\
\text { [40], The Nether- } \\
\text { lands }\end{array}$ & $\begin{array}{l}\text { Intervention: ACT-DL, } \\
\text { a mobile acceptance } \\
\text { and commitment thera- } \\
\text { py in daily life training } \\
\text { (smartphone) }\end{array}$ & $\begin{array}{l}\text { Sleep quality, ap- } \\
\text { praisal of the day, } \\
\text { affect (positive } \\
\text { and negative feel- } \\
\text { ings), cognition, } \\
\text { context (activity, } \\
\text { company and } \\
\text { whereabouts) }\end{array}$ & $\begin{array}{l}10 \text { times a day for } 3 \\
\text { days each week, for } 4 \\
\text { weeks }\end{array}$ & $\begin{array}{l}\text { Feasibility, accept- } \\
\text { ability, and effec- } \\
\text { tiveness of ACT- } \\
\text { DL }\left(\mathrm{EMI}^{\mathrm{g}}\right)\end{array}$ & $\begin{array}{l}\text { Mixed methods: } \\
\text { intervention } \\
\text { study with } 2 \\
\text { groups (experi- } \\
\text { mental interven- } \\
\text { tion or outpatient } \\
\text { treatment) }\end{array}$ & $\begin{array}{l}\text { Patients with a } \\
\text { mental health dis- } \\
\text { order such as } \\
\text { anxiety, mood, } \\
\text { somatoform, or } \\
\text { substance disor- } \\
\text { ders: experimen- } \\
\text { tal intervention } \\
(\mathrm{n}=49), 35 \% \\
\text { male (n=17), } \\
\text { mean age } 45.7 \\
(\text { SD 10.0); } \\
\text { healthy individu- } \\
\text { als ( } \mathrm{n}=112), 55 \% \\
\text { male (n=62), } \\
\text { mean age } 47.5 \\
\text { (SD 12.4) }\end{array}$ \\
\hline $\begin{array}{l}\text { Jiang, } 2016 \text { [41], } \\
\text { US }\end{array}$ & $\begin{array}{l}\text { Monitoring: Pocket } \\
\text { Personal Assistant for } \\
\text { Training Health (Pocket } \\
\text { PATH), a health self- } \\
\text { monitoring application } \\
\text { (smartphone) }\end{array}$ & $\begin{array}{l}\text { Spirometry, tem- } \\
\text { perature, blood } \\
\text { pressure, pulse, } \\
\text { symptoms, } \\
\text { weight }\end{array}$ & $\begin{array}{l}12 \text { months posttrans- } \\
\text { plantation, no app use } \\
\text { criteria }\end{array}$ & $\begin{array}{l}\text { Acceptance and } \\
\text { use of Pocket } \\
\text { PATH }\end{array}$ & $\begin{array}{l}\text { Quantitative: } \\
\text { cross-sectional } \\
\text { correlational de- } \\
\text { sign with sec- } \\
\text { ondary analysis } \\
\text { of existing RCT } \\
\text { data }\end{array}$ & $\begin{array}{l}\text { Lung transplanta- } \\
\text { tion recipients } \\
\text { transferred to the } \\
\text { acute cardiotho- } \\
\text { racic unit ( } \mathrm{n}=96) \text {, } \\
51 \% \text { male } \\
(\mathrm{n}=49) \text {, mean age } \\
57 \text { (SD 14) }\end{array}$ \\
\hline $\begin{array}{l}\text { Naughton, } 2016 \\
\text { [42], UK }\end{array}$ & $\begin{array}{l}\text { Intervention: Q-sense, } \\
\text { a smoking cessation } \\
\text { mobile phone applica- } \\
\text { tion (smartphone) }\end{array}$ & $\begin{array}{l}\text { Smoking behav- } \\
\text { ior, psychological } \\
\text { context, situation- } \\
\text { al context }\end{array}$ & $\begin{array}{l}1 \text { month before until } 2 \\
\text { weeks after a preset quit } \\
\text { date }\end{array}$ & $\begin{array}{l}\text { Feasibility of Q- } \\
\text { sense (EMI) }\end{array}$ & $\begin{array}{l}\text { Mixed methods: } \\
\text { an explanatory } \\
\text { sequential mixed } \\
\text { methods design } \\
\text { with app log data } \\
\text { and semistruc- } \\
\text { tured interviews }\end{array}$ & $\begin{array}{l}\text { Adult smokers } \\
\text { willing to set a } \\
\text { quit date in the } \\
\text { period between } 1 \\
\text { week and } 1 \\
\text { month after inclu- } \\
\text { sion }(\mathrm{n}=15), 53 \% \\
\text { male }(\mathrm{n}=8) \text {, age } \\
\text { range } 18-45\end{array}$ \\
\hline $\begin{array}{l}\text { Timmerman, } \\
2016 \text { [43], The } \\
\text { Netherlands }\end{array}$ & $\begin{array}{l}\text { Monitoring: telehealth } \\
\text { care application with a } \\
\text { symptom monitoring } \\
\text { module and web-based } \\
\text { exercise module } \\
\text { (smartphone and com- } \\
\text { puter) }\end{array}$ & $\begin{array}{l}\text { Pain, fatigue, } \\
\text { dyspnea }\end{array}$ & $\begin{array}{l}3 \text { days a week during } 2 \\
\text { weeks presurgery, the } \\
\text { first month postsurgery, } \\
\text { and } 2 \text { weeks prior to the } \\
\text { doctor consultation at } 3 \\
\text { and } 6 \text { months post- } \\
\text { surgery }\end{array}$ & $\begin{array}{l}\text { Development and } \\
\text { usability of a multi- } \\
\text { modal ICT }{ }^{\text {- }} \text {-sup- } \\
\text { ported rehabilita- } \\
\text { tion program for } \\
\text { lung cancer }\end{array}$ & $\begin{array}{l}\text { Qualitative: user- } \\
\text { centered design } \\
\text { with interviews } \\
\text { and focus groups }\end{array}$ & $\begin{array}{l}\text { Patients with } \\
\operatorname{NSCLC}^{\mathrm{i}}(\mathrm{n}=10) \text {, } \\
30 \% \text { male }(\mathrm{n}=3) \text {, } \\
\text { mean age } 62(\mathrm{SD} \\
11)\end{array}$ \\
\hline $\begin{array}{l}\text { Burke, } 2017 \text { [44], } \\
\text { US }\end{array}$ & $\begin{array}{l}\text { Intervention: standard } \\
\text { behavioral intervention } \\
\text { for weight (smartphone) }\end{array}$ & Not specified & $\begin{array}{l}5 \text { times a day for } 12 \\
\text { months }\end{array}$ & $\begin{array}{l}\text { Lessons learned } \\
\text { from development } \\
\text { and implementa- } \\
\text { tion of an EMA } \\
\text { study, focusing on } \\
\text { the methods and } \\
\text { logistics of conduct- } \\
\text { ing an EMA study } \\
\text { and including } \\
\text { strategies to ensure } \\
\text { adequate adher- } \\
\text { ence to EMA } \\
\text { prompts }\end{array}$ & $\begin{array}{l}\text { Qualitative: sin- } \\
\text { gle-group, obser- } \\
\text { vational design }\end{array}$ & $\begin{array}{l}\text { Former partici- } \\
\text { pants of laborato- } \\
\text { ry weight loss } \\
\text { studies }(\mathrm{n}=133) \text {, } \\
9 \% \text { male }(\mathrm{n}=12) \text {, } \\
\text { mean age } 51.09 \\
(\mathrm{SD} 10.10)\end{array}$ \\
\hline
\end{tabular}


First author, year, e-Diary characteristics country vice)
Study characteristics
Purpose of use ${ }^{\mathrm{a}}(\mathrm{de}-$
Constructs mea- Frequency of use and Study aims sured

duration
Design and data collection
Sample: target population, number of participants, sex, age (years)

\begin{tabular}{|c|c|c|c|c|c|c|}
\hline $\begin{array}{l}\text { Crane, } 2017 \text { [45], } \\
\text { UK }\end{array}$ & $\begin{array}{l}\text { Monitoring: DrinkLess, } \\
\text { an application (smart- } \\
\text { phone) }\end{array}$ & $\begin{array}{l}\text { Consequences of } \\
\text { alcohol consump- } \\
\text { tion, mood, pro- } \\
\text { ductivity, clarity, } \\
\text { sleep quality }\end{array}$ & $\begin{array}{l}\text { Daily, at least } 2 \text { weeks, } \\
\text { no app use criteria }\end{array}$ & $\begin{array}{l}\text { Usability of Drink- } \\
\text { Less }\end{array}$ & $\begin{array}{l}\text { Qualitative: us- } \\
\text { ability studies } \\
\text { with think-aloud } \\
\text { procedure and } \\
\text { semistructured } \\
\text { interviews }\end{array}$ & $\begin{array}{l}\text { Healthy individu- } \\
\text { als ( } \mathrm{n}=12 \text { for both } \\
\text { studies), 50\% } \\
\text { male ( } \mathrm{n}=6 \text { ), mean } \\
\text { age } 42 \text { (first } \\
\text { study) and } 40 \\
\text { (second study) }\end{array}$ \\
\hline $\begin{array}{l}\text { Freyne, } 2017 \\
\text { [46], Australia }\end{array}$ & $\begin{array}{l}\text { Intervention: PMRP }{ }^{\mathrm{j}} \text {, a } \\
\text { behavioral-based mo- } \\
\text { bile weight manage- } \\
\text { ment program and appli- } \\
\text { cation (smartphone) }\end{array}$ & $\begin{array}{l}\text { Meal diary for } \\
\text { previous day, } \\
\text { current weight, } \\
\text { dietary intake, } \\
\text { update food diary }\end{array}$ & $\begin{array}{l}3 \text { times a day for an in- } \\
\text { tervention period of } 12 \\
\text { weeks, followed by an- } \\
\text { other } 12 \text {-week period }\end{array}$ & $\begin{array}{l}\text { Role of push notifi- } \\
\text { cations in persuad- } \\
\text { ing users to engage } \\
\text { with self-monitor- } \\
\text { ing tasks }\end{array}$ & $\begin{array}{l}\text { Quantitative: in- } \\
\text { tervention study } \\
\text { with app log data }\end{array}$ & $\begin{array}{l}\text { Overweight } \\
\text { adults }(\text { BMI }>25 \\
\left.\mathrm{kg} / \mathrm{m}^{2} ; \mathrm{n}=75\right), \\
27 \% \text { male } \\
(\mathrm{n}=20) \text {, mean age } \\
48.6\end{array}$ \\
\hline $\begin{array}{l}\text { Kreyenbuhl, } \\
2018 \text { [47], US }\end{array}$ & $\begin{array}{l}\text { Intervention: MedAc- } \\
\text { tive, an application } \\
\text { (smartphone) }\end{array}$ & $\begin{array}{l}\text { Medication adher- } \\
\text { ence, positive } \\
\text { psychotic symp- } \\
\text { toms, medication } \\
\text { side effects }\end{array}$ & Daily for 2 weeks & $\begin{array}{l}\text { Acceptability and } \\
\text { feasibility of } \\
\text { MedActive (EMA) }\end{array}$ & $\begin{array}{l}\text { Quantitative: us- } \\
\text { er-centered de- } \\
\text { sign with surveys }\end{array}$ & $\begin{array}{l}\text { Patients with } \\
\text { schizophrenia } \\
\text { spectrum disorder } \\
\text { taking } \geq 1 \text { oral an- } \\
\text { tipsychotic medi- } \\
\text { cations ( } \mathrm{n}=7 \text { ), } \\
100 \% \text { male } \\
(\mathrm{n}=7) \text {, mean age } \\
47.6 \text { (SD 10.4) }\end{array}$ \\
\hline $\begin{array}{l}\text { Liu, 2018, US } \\
\text { [48] }\end{array}$ & $\begin{array}{l}\text { Monitoring: LoseIt, a } \\
\text { physical activity and } \\
\text { diet tracking application } \\
\text { (smartphone) }\end{array}$ & Food intake & $\begin{array}{l}\text { At least } 3 \text { days a week } \\
\text { for } 2 \text { weeks }\end{array}$ & $\begin{array}{l}\text { Effectiveness of } \\
\text { LoseIt }\end{array}$ & $\begin{array}{l}\text { Quantitative: ran- } \\
\text { domized trial } \\
\text { with } 2 \text { groups } \\
\text { (goal setting re- } \\
\text { minders or gener- } \\
\text { ic reminders) } \\
\text { with pre- and } \\
\text { posttests }\end{array}$ & $\begin{array}{l}\text { College students } \\
(\mathrm{n}=50), 38 \% \\
\text { male }(\mathrm{n}=19), \\
\text { mean age } 21(\mathrm{SD} \\
1.8)\end{array}$ \\
\hline $\begin{array}{l}\text { Tomko, } 2018 \\
\text { [49], US }\end{array}$ & $\begin{array}{l}\text { Monitoring: REDCap, } \\
\text { ambulatory assessment } \\
\text { software (computer) }\end{array}$ & $\begin{array}{l}\text { Smoking, sub- } \\
\text { stance use, medi- } \\
\text { cation adherence }\end{array}$ & $\begin{array}{l}3 \text { times daily for } 8 \\
\text { weeks }\end{array}$ & $\begin{array}{l}\text { Feasibility of ambu- } \\
\text { latory assessment } \\
\text { (here applied in } \\
\text { smoking cessation) } \\
\text { for research purpos- } \\
\text { es (EMA) }\end{array}$ & $\begin{array}{l}\text { Quantitative: fea- } \\
\text { sibility study } \\
\text { within a double- } \\
\text { blind RCT with } 2 \\
\text { groups ( } \mathrm{N} \text {-acetyl- } \\
\text { cysteine or place- } \\
\text { bo) }\end{array}$ & $\begin{array}{l}\text { Adult smokers } \\
(\mathrm{n}=36), 50 \% \\
\text { male }(\mathrm{n}=18), \\
\text { mean age } 41.1 \\
(\mathrm{SD} 12.7)\end{array}$ \\
\hline
\end{tabular}

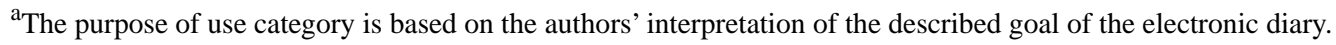

bEMA: ecological momentary assessment.

${ }^{\mathrm{c}} \mathrm{RCT}$ : randomized controlled trial.

${ }^{\mathrm{d}} \mathrm{CBT}$ : cognitive behavioral therapy.

${ }^{\mathrm{e}}$ TMD: temporomandibular disorder.

${ }^{\mathrm{f}}$ IT: information technology.

${ }^{\mathrm{g}}$ EMI: ecological momentary intervention.

hICT: information communication technology.

${ }^{\mathrm{i}}$ NSCLC: non-small cell lung cancer.

${ }^{\mathrm{j}}$ PMRP: partial meal replacement program.
}

The factors that influence the use of electronic diaries in health care were not the primary aim in all included studies. These factors were mentioned as part of a larger study, such as a randomized controlled trial or an intervention study. Studies focused on usability in half of the articles (10/22, 45\%), followed by feasibility and effectiveness $(7 / 22,32 \%)$ and development $(5 / 22,23 \%)$. The design of these studies was quantitative $(11 / 22,50 \%)$, mixed $(6 / 22,27 \%)$, or qualitative $(5 / 22,23 \%)$. The number of participants ranged from 3 to 348 , with a mean age of 49 years. Of these, 37.0\% (493/1341) were male. The majority of the studies included patients with physical symptoms $(12 / 22,55 \%)$, whereas healthy individuals $(7 / 22$, 
$32 \%)$ and patients with mental health symptoms $(3 / 22,13 \%)$ were less often described.

\section{Factors That Influence the Use of Electronic Diaries}

The CFIR [25] was used to perform the qualitative thematic analysis of the factors that influence the use of electronic diaries in health care. The results of this qualitative thematic analysis were organized along 3 CFIR categories: intervention [29-31,33-49], user characteristics $[28,32,36,37,39,41,42,44,45,49], \quad$ and process [30-33,38,41,43-45,47,49]. No results were found for the 2 other CFIR categories: inner setting and outer setting. Figure 2 gives an overview of these categories, themes, and subthemes.

Figure 2. Visual representation of the factors that influence the use of electronic diaries in health care.

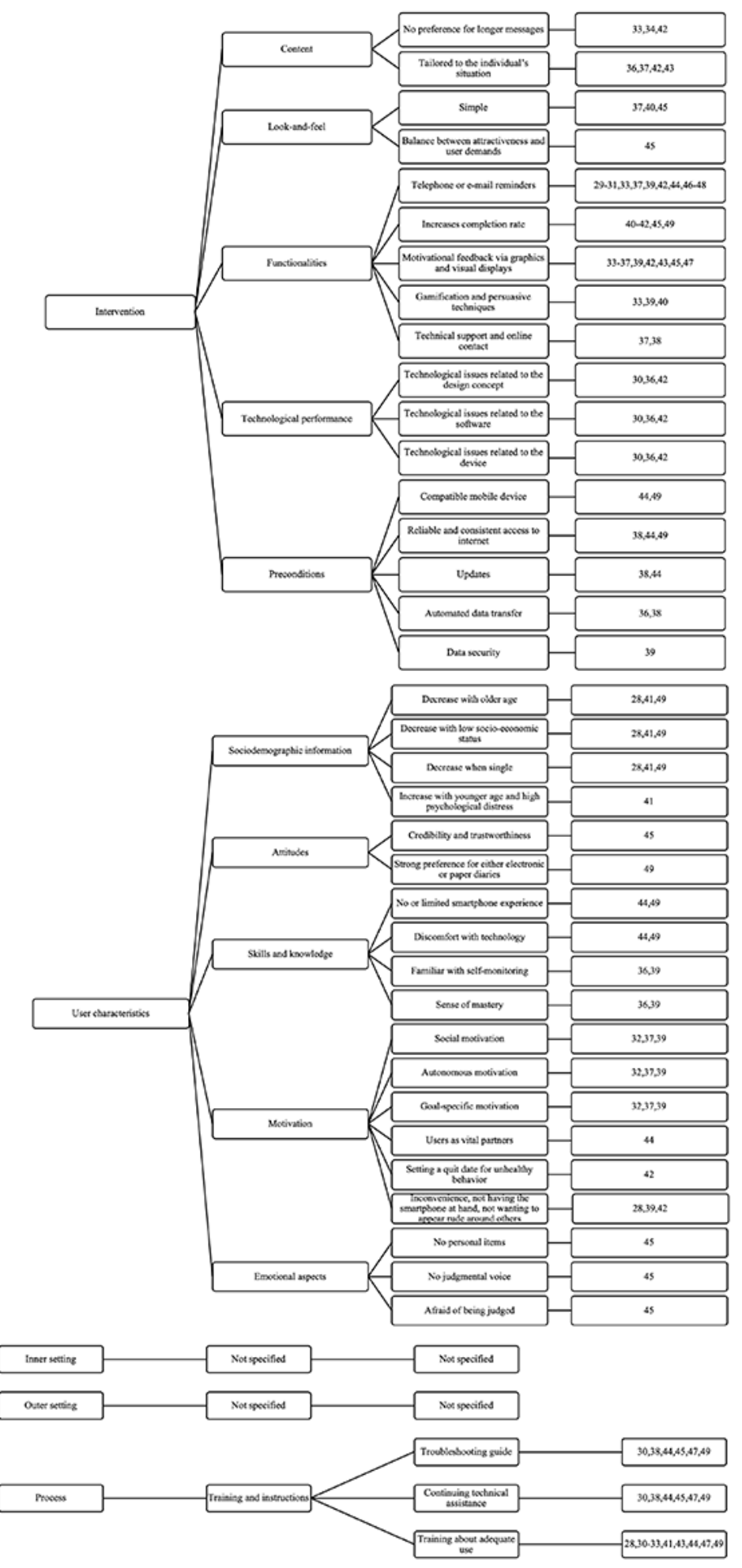




\section{Intervention}

The first category describes the key attributes of an electronic diary device, a smartphone application, or a web-based module. Five themes specify the intervention.

The first theme, "content," refers to the information in an electronic diary. Smartphone applications and web-based modules consisted of several content types like EMA, reminders, and reward messages [33,40,45,47-49]. This content supports communication between the patient and the health care professional. Long messages are considered too time-consuming to read, and users would therefore skip screens $[33,34,42]$. Furthermore, users may prefer both cartoons or videos and text [40-42,45,49]. Moreover, diary questions should be tailored to the individual's situation $[36,37,42,43]$. Users are inconclusive about the scope of the constructs measured; some may prefer an exclusive focus on one topic, whereas others may find that too limited $[37,47]$.

The second theme, "look and feel," refers to the configuration or layout of an electronic diary. The user interface should be both simple and attractive [37,40,45]. However, a balance between attractiveness and user demands is required. Users may prefer a visually appealing user interface with minimal demands on them [45].

The third theme, "functionalities," refers to the activities that a user can perform within an application, ranging from procedures for recording and uploading data to customization of the user interface. Telephone or email reminders, either programmable or automated, notify the user to complete a questionnaire, which increases the completion rate [29-31,33,37,39,42,44,46-48]. Furthermore, manually entering several indicators per day increases participant burden [40-42,45,49]. Moreover, users want to receive motivational feedback about their results via clear graphics and visual displays [33-37,39,42,43,45,47]. Gamification and persuasive techniques can be used to provide motivational feedback to increase completion rates $[33,39,40]$. Additionally, Tang et al [37] and Triantafyllidis et al [38] identified that technical support and online contact with, for example, a health care professional increase the use of an electronic diary.

The fourth theme, "technological performance," refers to the technological issues that users encounter while using an electronic diary. Users can experience technological issues related to the design concept (eg, navigation problems), the software, or the device (eg, battery attrition). These errors reduce the usability of an electronic diary [30,36,42].

The fifth theme, "preconditions," refers to the conditions that must be fulfilled before a smartphone application or a web-based module can function properly. Burke et al [44] and Tomko et al [49] suggested that users are provided with a compatible mobile device (with sufficient memory, processing speed, and a functioning camera) to overcome the barrier of installing additional hardware or software on the user's device. Moreover, Burke et al [44], Tomko et al [49], and Triantafyllidis et al [38] stated that users need reliable and consistent access to the internet while using the tool. Furthermore, they suggested checking for operating system and other smartphone updates that potentially interfere with the smartphone application of interest $[38,44]$. The electronic diary should be updated continuously; hence, bandwidth limitations should be taken into account, especially for web-based modules [31]. Automated data transfer to the background server or another device must be seamless for the individual to be able to use the device with minimal effort $[36,38]$. Depending on the type of data, users highly value data security. They are especially concerned that data would not be shared with health insurers [39].

\section{User Characteristics}

The second category describes the characteristics of the individuals who use the electronic diary, in this case, healthy individuals and patients with physical or psychosocial problems. Five themes specify the user characteristics.

The first theme, "sociodemographic information," refers to the characteristics of a population such as gender, age, and marital status. The use of an electronic diary decreases when individuals are older, have a low socioeconomic status, or are unmarried, separated, divorced, or widowed [28,41,49], whereas an increase in the use of these tools is seen when individuals experience high psychological distress [41].

The second theme, "attitudes," refers to the way a user feels and behaves with regard to an electronic diary. Crane et al [45] concluded that users' positive attitudes towards smartphone applications or web-based modules are based on credibility and trustworthiness of the information. Moreover, Tomko et al [49] stated that users may have strong preferences for either electronic or paper diaries.

The third theme, "skills and knowledge," refers to the information that a user has about electronic diaries and the ability to use these tools. Users with no or limited smartphone experience and who experience discomfort with technology will not use electronic diaries adequately. Extra staff is required to train these users [44,49]. Additionally, users who become familiar with self-monitoring or get a sense of mastery over their problems will lose their motivation and consequently stop or reduce their app use [36,39].

The fourth theme, "motivation," refers to the needs, desires, and drives of the individual to use an electronic diary. Naughton et al [42], Anderson et al [39], and Aaron et al [28] stated that missing data are not caused by low motivation, but by discomfort, not having the smartphone at hand, or not wanting to appear rude around others. Social motivation, autonomous motivation, and goal-specific motivation increase the adherence to using electronic diaries [32,37,39]. Furthermore, making users vital partners in the development of an electronic diary keeps them motivated to use these devices [44]. In case of unhealthy behaviors, setting a quit date boosts users' commitment [42].

The fifth theme, "emotional aspects," refers to the feelings that are induced by using an electronic diary. When diary questions are too personal or judgmental, users are less likely to engage with a smartphone application or a web-based module [45]. Furthermore, they want to keep their data private because they are afraid of being judged [45]. However, in the study by Aaron et al [28], emotional aspects were the least mentioned reasons 
for missing a questionnaire, although Crane et al [45] found that users feel guilty when diaries are missed.

\section{Process}

The third category describes the activities related to the implementation process. One theme specifies the process.

The theme, "training and instructions," refers to how users are guided and instructed to adequately use an electronic diary. Training (eg, face-to-face group kick-off presentation, training session to familiarize with the tool and troubleshoot issues) could result in higher use of these tools [28,30-33,41,43,44,47,49]. Furthermore, users may prefer a troubleshooting guide with step-by-step instructions or continuing technical assistance in case of technological issues from the staff or development team [30,38,44,45,47,49].

\section{Discussion}

\section{Principal Findings}

This scoping review maps the existing knowledge and gaps concerning factors that influence the use of electronic diaries in health care. Due to technological developments in the last decades, electronic diaries have become increasingly available and popular in research and routine clinical practice. This increased interest is also visible in the large number of articles published between 2000 and 2018. However, only a small number of these articles focused on factors that influence the use of electronic diaries. Additionally, an even smaller number of the selected articles focused on implementing these tools in daily clinical practice.

In this scoping review, 22 articles were selected based on the predefined eligibility criteria. For the categories of intervention, user characteristics, and process of the CFIR [25], 11 themes were identified, whereas no empirical data were found for the 2 other CFIR categories: inner setting and outer setting. The use of an electronic diary is facilitated when it is a visually appealing tool with various content types, including reminders, clear in-app data visualizations tailored to the individual, and minimal user demands to increase the user's engagement. A compatible mobile device with reliable internet access and automated data transfer supports adequate use of an electronic diary. Additionally, the user needs to have smartphone experience, intrinsic motivation, and a clear rationale to monitor one's own behavior. Finally, both theoretical training and practical training are recommended to foster the implementation process. However, the required content and procedures of such training were not described in the included studies.

Based on these results and considering relevant implementation and adoption models, 2 findings attract attention. First, it is remarkable that there were only empirical data about the influence of the characteristics of the electronic diary, the individual, and the implementation process, whereas the CFIR and other implementation frameworks also emphasize the importance of factors related to the organization in which the care is provided or the organizational culture (inner setting) and the competition or the pressure from external partners and the regulations or legislation concerning electronic diaries in clinical practice (outer setting) [25]. Recent research on the implementation of patient-reported outcome measures also highlights the importance of investing sufficient time and resources to support health care professionals [50-54].

Second, the scope of the implementation framework CFIR, used in this review, appears to be wider than adoption models that are traditionally used to evaluate user engagement and continued use of information systems and mobile technologies, like the Technology Acceptance Model [55-59]. The adoption models limit the scope to characteristics of the electronic diary and the individual user, whereas the CFIR also takes into account the process of implementation in daily clinical practice. In this review, the importance of training and instructions was revealed. The importance of hands-on instructions (individual coaching on the job sessions to familiarize with the use of experience-sampling technology in daily clinical practice, using real-world examples) as well as the ability to contact a help desk in case of practical and technological issues was underlined in our previous study as well [27]. Also, regarding the characteristics of the electronic diary, the adoption models have a smaller focus. They only highlight the running software as a contributing factor, while this scoping review identified that the information about and the layout of these diaries, as well as the technological issues and preconditions, also influence their use [55-59]. However, when considering the characteristics of the individual user, this scoping review revealed personal characteristics such as age, along with attitudes, emotions, and behaviors, while adoption models also focus on social influence and self-efficacy as contributing factors [55-59].

Implementation literature emphasizes that attention should be paid to the range of influencing factors to achieve a successful implementation in daily clinical practice [25,50-54]. Consequently, sustainable use of electronic diaries requires that health care organizations or professionals not only direct attention towards software, hardware, and the target population of the tool but also to the economic and political organizational context, the innovation climate in the organization, and the embedding of the tool in routine clinical practice.

\section{Strengths and Limitations}

Several limitations have to be kept in mind while interpreting the results of this scoping review. The structured literature search was based on a combination of key words defined by preliminary literature exploration and expert consultation. Despite a broad search approach, it is still possible that articles were missed since the research topic was often not the primary aim of the included studies. This possibly resulted in selection bias. However, the additional hand search minimized this potential shortcoming. It is also worth noting that most of the articles were excluded based on title screening. This can be seen as a limitation, but we think this approach is justifiable in our sensitive search. We performed an iterative screening process that required the researchers to engage in a reflexive way and repeat steps to ensure that the literature was covered in an extensive way. When the relevance of the study was not clear from the title, the abstract was always read. But it is still possible that we missed some articles. Moreover, as an extra check on the 3 -step screening process, we read the full texts of a random sample of 50 titles and 50 abstracts. In only 4 articles, we found 
information in the results or the discussion related to our scope. Furthermore, as the aim of this scoping review was to map the existing empirical knowledge and identify any gaps about factors that influence the use of electronic diaries in health care, no study quality assessment was performed. Moreover, a scoping review does not endeavor to give a summary of the existing literature or compare results (in contrast to a systematic review of, for example, randomized controlled trials on efficacy). Therefore, we did not intend to draw firm conclusions regarding useful and effective features of electronic diaries based on quantified outcomes. We provide, to our knowledge, a first overview of the factors that influence the use of electronic diaries in health care. Future research with longitudinal or mixed methods study designs should focus on the causal relationships between the influencing factors and the use of electronic diaries in health care in order to get a deeper understanding of the causality. Also, a quite diverse sample of studies was included. However, we are convinced that we have achieved the scope of interest of this scoping review. We looked in more detail at similarities and differences in the results of the included studies, based on the purpose of use (monitoring versus intervention), target population (healthy individuals versus patients), setting, study aims, and design (feasibility versus usability versus development). However, we concluded that this synthesis cannot be performed based on the results of the information found in this scoping review. More research is needed in this field.
Additionally, the structured literature search was restricted to peer-reviewed databases and so, empirical research. Book chapters and grey literature were not included, which means that additional empirical data can be lacking. This scoping review has several methodological strengths as well. First, a systematic approach was used based on the methodological framework by Arksey and O'Malley [24]. The interprofessional nature of the research team extended the scope of this review, and the consultation of 2 experts in the field validated the search terms. Furthermore, the 3-step screening process was consistently performed by 2 researchers. Second, the thematic analysis organized according to an implementation research perspective led to a synthesis contributing to future understanding of the implementation of electronic diaries in health care.

\section{Conclusion}

This scoping review demonstrates that the use of electronic diaries may be influenced by characteristics of the electronic diary, the individual user, and the implementation process. However, the number of empirical studies on the topic was limited. Studies that take into account the setting in which to implement the diaries, such as the organizational context, the implementation climate, and available organizational resources, were lacking. Future research should focus on these factors and on the causal relationships between the different factors to investigate the continued use of these innovative tools.

\section{Acknowledgments}

We thank Gaston Jamin and François Engelen for sharing their ideas about key authors and key publications on the topic of interest.

This project was supported by the Brightlands Innovation Program LIME (Limburg Measures; limeconnect.nl), a program that facilitates smarter measurement methods and more efficient data collection for better care and health; the Province of Limburg; Zuyd University of Applied Sciences; and Maastricht University, the Netherlands.

\section{Conflicts of Interest}

None declared.

\section{Multimedia Appendix 1}

Table S1. Patterns of publications. [DOCX File, 13 KB-Multimedia Appendix 1]

\section{References}

1. Shindorf Z. Exploring Barriers to the Generalization of Social Skills Interventions for Children Diagnosed with ASD: A Qualitative Analysis of "Youth Engagement Through Intervention". University of Montana. 2016. URL: https://scholarworks. umt.edu/etd/10911/ [accessed 2019-09-10]

2. Smith PC, Araya-Guerra R, Bublitz C, Parnes B, Dickinson LM, Van Vorst R, et al. Missing clinical information during primary care visits. JAMA 2005 Feb 02;293(5):565-571. [doi: 10.1001/jama.293.5.565] [Medline: 15687311]

3. Higgs J, Jones M, Loftus S, Christensen N, editors. Clinical reasoning in the health professions, fourth edition. Amsterdam, Netherlands: Elsevier; 2018.

4. van Os J, Delespaul P, Wigman J, Myin-Germeys I, Wichers M. Beyond DSM and ICD: introducing "precision diagnosis" for psychiatry using momentary assessment technology. World Psychiatry 2013 Jun;12(2):113-117 [FREE Full text] [doi: 10.1002/wps.20046] [Medline: 23737412]

5. van Os J, Lataster T, Delespaul P, Wichers M, Myin-Germeys I. Evidence that a psychopathology interactome has diagnostic value, predicting clinical needs: an experience sampling study. PLoS One 2014;9(1):e86652 [FREE Full text] [doi: 10.1371/journal.pone.0086652] [Medline: 24466189] 
6. National Research Council. Cognitive Aspects of Survey Methodology: Building a Bridge Between Disciplines. In: National Academies 030907. Washington, DC: The National Academies Press; 1984.

7. Stone A, Bachrach C, Jobe J, Kurtzman H, Cain V, editors. The science of self-report: Implications for research and practice. New York, NY: Psychology Press; 2000.

8. Stone AA, Shiffman S, Schwartz JE, Broderick JE, Hufford MR. Patient non-compliance with paper diaries. BMJ 2002 May 18;324(7347):1193-1194 [FREE Full text] [doi: 10.1136/bmj.324.7347.1193] [Medline: 12016186]

9. Byrom B, Tiplady B, editors. ePRO: electronic solutions for patient-reported data. Abingdon-on-Thames, Oxfordshire, England: Routledge; 2010.

10. Dale O, Hagen KB. Despite technical problems personal digital assistants outperform pen and paper when collecting patient diary data. J Clin Epidemiol 2007 Jan;60(1):8-17. [doi: 10.1016/j.jclinepi.2006.04.005] [Medline: 17161749]

11. Milligan C, Bingley A, Gatrell A. Digging deep: using diary techniques to explore the place of health and well-being amongst older people. Soc Sci Med 2005 Nov;61(9):1882-1892. [doi: 10.1016/j.socscimed.2005.04.002] [Medline: 15913863]

12. Ladores S, Bray LA. Electronic Diaries in Healthcare: A Review of Literature. JNPARR 2009:13-21. [doi: 10.13178/jnparr.2017.0702.0705]

13. Green AS, Rafaeli E, Bolger N, Shrout PE, Reis HT. Paper or plastic? Data equivalence in paper and electronic diaries. Psychol Methods 2006 Mar;11(1):87-105. [doi: 10.1037/1082-989X.11.1.87] [Medline: 16594769]

14. Stone AA, Shiffman S, Schwartz JE, Broderick JE, Hufford MR. Patient compliance with paper and electronic diaries. Control Clin Trials 2003 Apr;24(2):182-199. [doi: 10.1016/s0197-2456(02)00320-3] [Medline: 12689739]

15. Bolger N, Davis A, Rafaeli E. Diary methods: capturing life as it is lived. Annu Rev Psychol 2003;54:579-616. [doi: 10.1146/annurev.psych.54.101601.145030] [Medline: 12499517]

16. Drummond HE, Ghosh S, Ferguson A, Brackenridge D, Tiplady B. Electronic quality of life questionnaires: a comparison of pen-based electronic questionnaires with conventional paper in a gastrointestinal study. Qual Life Res 1995 Feb;4(1):21-26. [doi: 10.1007/BF00434379] [Medline: 7711686]

17. Finkelstein J, Cabrera MR, Hripcsak G. Internet-based home asthma telemonitoring: can patients handle the technology? Chest 2000 Jan;117(1):148-155. [doi: 10.1378/chest.117.1.148] [Medline: 10631213 ]

18. Hufford M, Shields A. Electronic diaries: Applications and What Works in the Field. Applied Clinical Trials 2002:46-59.

19. Totterdell P, Folkard S. In situ repeated measures of affect and cognitive performance facilitated by use of a hand-held computer. Behavior Research Methods, Instruments, \& Computers 1992 Dec;24(4):545-553. [doi: 10.3758/bf03203603]

20. Kamarck TW, Shiffman SM, Smithline L, Goodie JL, Paty JA, Gnys M, et al. Effects of task strain, social conflict, and emotional activation on ambulatory cardiovascular activity: daily life consequences of recurring stress in a multiethnic adult sample. Health Psychol 1998 Jan;17(1):17-29. [doi: 10.1037//0278-6133.17.1.17] [Medline: 9459066]

21. Hsiao C, Chang J, Tang K. Exploring the influential factors in continuance usage of mobile social Apps: Satisfaction, habit, and customer value perspectives. Telematics and Informatics 2016 May;33(2):342-355. [doi: 10.1016/j.tele.2015.08.014]

22. Kang S. Factors influencing intention of mobile application use. IJMC 2014;12(4):360. [doi: 10.1504/ijmc.2014.063653]

23. Wang BR, Park J, Chung K, Choi IY. Influential Factors of Smart Health Users according to Usage Experience and Intention to Use. Wireless Pers Commun 2014 Apr 24;79(4):2671-2683. [doi: 10.1007/s11277-014-1769-0]

24. Arksey H, O'Malley L. Scoping studies: towards a methodological framework. International Journal of Social Research Methodology 2005 Feb;8(1):19-32. [doi: 10.1080/1364557032000119616]

25. Damschroder LJ, Aron DC, Keith RE, Kirsh SR, Alexander JA, Lowery JC. Fostering implementation of health services research findings into practice: a consolidated framework for advancing implementation science. Implement Sci 2009 Aug 07;4:50 [FREE Full text] [doi: 10.1186/1748-5908-4-50] [Medline: 19664226]

26. Hsieh H, Shannon SE. Three approaches to qualitative content analysis. Qual Health Res 2005 Nov;15(9):1277-1288. [doi: 10.1177/1049732305276687] [Medline: 16204405]

27. Daniëls NEM, Hochstenbach LMJ, van Bokhoven MA, Beurskens AJHM, Delespaul PAEG. Implementing Experience Sampling Technology for Functional Analysis in Family Medicine - A Design Thinking Approach. Front Psychol 2019 Dec 11;10:2782 [FREE Full text] [doi: 10.3389/fpsyg.2019.02782] [Medline: $\underline{31920830]}$

28. Aaron LA, Mancl L, Turner JA, Sawchuk CN, Klein KM. Reasons for missing interviews in the daily electronic assessment of pain, mood, and stress. Pain 2004 Jun;109(3):389-398. [doi: 10.1016/j.pain.2004.02.014] [Medline: 15157700]

29. Litcher-Kelly L, Kellerman Q, Hanauer SB, Stone AA. Feasibility and utility of an electronic diary to assess self-report symptoms in patients with inflammatory bowel disease. Ann Behav Med 2007 Apr;33(2):207-212. [doi: 10.1007/BF02879902] [Medline: 17447873 ]

30. Welch J, Dowell S, Johnson C. Feasibility of using a personal digital assistant to self-monitor diet and fluid intake: a pilot study. Nephrol Nurs J 2007;34(1):43-8; quiz 49. [Medline: 17345691]

31. Stevens VJ, Funk KL, Brantley PJ, Erlinger TP, Myers VH, Champagne CM, et al. Design and implementation of an interactive website to support long-term maintenance of weight loss. J Med Internet Res 2008 Jan 25;10(1):e1 [FREE Full text] [doi: 10.2196/jmir.931] [Medline: 18244892]

32. Webber KH, Tate DF, Ward DS, Bowling JM. Motivation and its relationship to adherence to self-monitoring and weight loss in a 16-week Internet behavioral weight loss intervention. J Nutr Educ Behav 2010;42(3):161-167. [doi: 10.1016/j.jneb.2009.03.001] [Medline: 20138583] 
33. Ahtinen A, Mattila E, Välkkynen P, Kaipainen K, Vanhala T, Ermes M, et al. Mobile mental wellness training for stress management: feasibility and design implications based on a one-month field study. JMIR Mhealth Uhealth 2013 Jul 10;1(2):e11 [FREE Full text] [doi: 10.2196/mhealth.2596] [Medline: 25100683]

34. Ben-Zeev D, Kaiser SM, Brenner CJ, Begale M, Duffecy J, Mohr DC. Development and usability testing of FOCUS: a smartphone system for self-management of schizophrenia. Psychiatr Rehabil J 2013 Dec;36(4):289-296 [FREE Full text] [doi: 10.1037/prj0000019] [Medline: 24015913]

35. Ma J, Xiao L, Blonstein AC. Measurement of self-monitoring web technology acceptance and use in an e-health weight-loss trial. Telemed J E Health 2013 Oct;19(10):739-745 [FREE Full text] [doi: 10.1089/tmj.2013.0009] [Medline: 23952787]

36. Tatara N, Arsand E, Skrøvseth SO, Hartvigsen G. Long-term engagement with a mobile self-management system for people with type 2 diabetes. JMIR Mhealth Uhealth 2013 Mar 27;1(1):e1 [FREE Full text] [doi: 10.2196/mhealth.2432] [Medline: 25100649]

37. Tang J, Abraham C, Stamp E, Greaves C. How can weight-loss app designers' best engage and support users? A qualitative investigation. Br J Health Psychol 2015 Feb;20(1):151-171. [doi: 10.1111/bjhp.12114] [Medline: 25130682]

38. Triantafyllidis A, Velardo C, Chantler T, Shah SA, Paton C, Khorshidi R, SUPPORT-HF Investigators. A personalised mobile-based home monitoring system for heart failure: The SUPPORT-HF Study. Int J Med Inform 2015 Oct;84(10):743-753. [doi: 10.1016/j.ijmedinf.2015.05.003] [Medline: 26037921]

39. Anderson K, Burford O, Emmerton L. Mobile Health Apps to Facilitate Self-Care: A Qualitative Study of User Experiences. PLoS One 2016;11(5):e0156164 [FREE Full text] [doi: 10.1371/journal.pone.0156164] [Medline: 27214203]

40. Batink T, Bakker J, Vaessen T, Kasanova Z, Collip D, van Os J, et al. Acceptance and Commitment Therapy in Daily Life Training: A Feasibility Study of an mHealth Intervention. JMIR Mhealth Uhealth 2016 Sep 15;4(3):e103 [FREE Full text] [doi: 10.2196/mhealth.5437] [Medline: 27634747]

41. Jiang Y, Sereika SM, Dabbs AD, Handler SM, Schlenk EA. Acceptance and Use of Mobile Technology for Health Self-Monitoring in Lung Transplant Recipients during the First Year Post-Transplantation. Appl Clin Inform 2016;7(2):430-445 [FREE Full text] [doi: 10.4338/ACI-2015-12-RA-0170] [Medline: 27437052]

42. Naughton F, Hopewell S, Lathia N, Schalbroeck R, Brown C, Mascolo C, et al. A Context-Sensing Mobile Phone App (Q Sense) for Smoking Cessation: A Mixed-Methods Study. JMIR Mhealth Uhealth 2016 Sep 16;4(3):e106 [FREE Full text] [doi: 10.2196/mhealth.5787] [Medline: 27637405]

43. Timmerman JG, Tönis TM, Dekker-van Weering MGH, Stuiver MM, Wouters MWJM, van Harten WH, et al. Co-creation of an ICT-supported cancer rehabilitation application for resected lung cancer survivors: design and evaluation. BMC Health Serv Res 2016 Apr 27;16:155 [FREE Full text] [doi: 10.1186/s12913-016-1385-7] [Medline: 27121869]

44. Burke LE, Shiffman S, Music E, Styn MA, Kriska A, Smailagic A, et al. Ecological Momentary Assessment in Behavioral Research: Addressing Technological and Human Participant Challenges. J Med Internet Res 2017 Mar 15;19(3):e77 [FREE Full text] [doi: 10.2196/jmir.7138] [Medline: 28298264]

45. Crane D, Garnett C, Brown J, West R, Michie S. Factors Influencing Usability of a Smartphone App to Reduce Excessive Alcohol Consumption: Think Aloud and Interview Studies. Front Public Health 2017;5:39 [FREE Full text] [doi: 10.3389/fpubh.2017.00039] [Medline: 28421175]

46. Freyne J, Yin J, Brindal E, Hendrie GA, Berkovsky S, Noakes M. Push Notifications in Diet Apps: Influencing Engagement Times and Tasks. International Journal of Human-Computer Interaction 2017 Jan 11;33(10):833-845. [doi:

$\underline{10.1080 / 10447318.2017 .1278896]}$

47. Kreyenbuhl J, Record EJ, Himelhoch S, Charlotte M, Palmer-Bacon J, Dixon LB, et al. Development and Feasibility Testing of a Smartphone Intervention to Improve Adherence to Antipsychotic Medications. Clin Schizophr Relat Psychoses 2019;12(4):152-167 [FREE Full text] [doi: 10.3371/CSRP.KRRE.070816] [Medline: 27454213]

48. Liu S, Willoughby JF. Do Fitness Apps Need Text Reminders? An Experiment Testing Goal-Setting Text Message Reminders to Promote Self-Monitoring. J Health Commun 2018;23(4):379-386. [doi: 10.1080/10810730.2018.1455768] [Medline: 29601270]

49. Tomko RL, Gray KM, Oppenheimer SR, Wahlquist AE, McClure EA. Using REDCap for ambulatory assessment: Implementation in a clinical trial for smoking cessation to augment in-person data collection. Am J Drug Alcohol Abuse 2018;45(1):26-41 [FREE Full text] [doi: 10.1080/00952990.2018.1437445] [Medline: 29513609]

50. Antunes B, Harding R, Higginson IJ, EUROIMPACT. Implementing patient-reported outcome measures in palliative care clinical practice: a systematic review of facilitators and barriers. Palliat Med 2014 Feb;28(2):158-175 [FREE Full text] [doi: 10.1177/0269216313491619] [Medline: 23801463]

51. Boyce MB, Browne JP, Greenhalgh J. The experiences of professionals with using information from patient-reported outcome measures to improve the quality of healthcare: a systematic review of qualitative research. BMJ Qual Saf 2014 Jun;23(6):508-518. [doi: 10.1136/bmjqs-2013-002524] [Medline: 24505110]

52. Duncan EA, Murray J. The barriers and facilitators to routine outcome measurement by allied health professionals in practice: a systematic review. BMC Health Serv Res 2012 May 22;12(1):96 [FREE Full text] [doi: 10.1186/1472-6963-12-96] [Medline: 22506982] 
53. Greenhalgh J, Dalkin S, Gooding K, Gibbons E, Wright J, Meads D, et al. Functionality and feedback: a realist synthesis of the collation, interpretation and utilisation of patient-reported outcome measures data to improve patient care. Health Serv Deliv Res 2017 Jan;5(2):1-280. [doi: 10.3310/hsdr05020] [Medline: 28121094]

54. Howell D, Molloy S, Wilkinson K, Green E, Orchard K, Wang K, et al. Patient-reported outcomes in routine cancer clinical practice: a scoping review of use, impact on health outcomes, and implementation factors. Ann Oncol 2015

Sep;26(9):1846-1858 [FREE Full text] [doi: 10.1093/annonc/mdv181] [Medline: 25888610]

55. Rogers EM. Diffusion of Innovations: modifications of a model for telecommunications. In: Stoetzer MW, Mahler A, editors. Die diffusion von innovationen in der telekommunikation. Schriftenreihe des Wissenschaftlichen Instituts für Kommunikationsdienste, vol 17. Berlin, Heidelberg: Springer; 1995.

56. Ajzen I. From Intentions to Actions: A Theory of Planned Behavior. In: Kuhl J, Beckmann J, editors. Action Control. SSSP Springer Series in Social Psychology. Berlin, Heidelberg: Springer; 1985:11-39.

57. Davis FD. Perceived Usefulness, Perceived Ease of Use, and User Acceptance of Information Technology. MIS Quarterly 1989 Sep;13(3):319-340. [doi: 10.2307/249008]

58. Bandura A. Self-Efficacy: The exercise of control. New York, NY: Worth Publishers; 1997.

59. Venkatesh V, Morris MG, Davis GB, Davis FD. User Acceptance of Information Technology: Toward a Unified View. MIS Quarterly 2003;27(3):425-478. [doi: 10.2307/30036540]

\begin{abstract}
Abbreviations
CFIR: Consolidated Framework for Implementation Research

EMA: ecological momentary assessment
\end{abstract}

Edited by L Buis; submitted 22.04.20; peer-reviewed by A Triantafyllidis, D Salvi, M Dunbar, J Willoughby; comments to author
04.09.20; revised version received 30.10.20; accepted 25.02.21; published 01.06.21
Please cite as:
Daniëls NEM, Hochstenbach LMJ, van Zelst C, van Bokhoven MA, Delespaul PAEG, Beurskens AJHM
Factors That Influence the Use of Electronic Diaries in Health Care: Scoping Review
JMIR Mhealth Uhealth 2021;9(6):e19536
URL: $\underline{\text { https://mhealth.jmir.org/2021/6/e19536 }}$
doi: $\underline{10.2196 / 19536}$
PMID:

CNaomi E M Daniëls, Laura M J Hochstenbach, Catherine van Zelst, Marloes A van Bokhoven, Philippe A E G Delespaul, Anna J H M Beurskens. Originally published in JMIR mHealth and uHealth (https://mhealth.jmir.org), 01.06.2021. This is an open-access article distributed under the terms of the Creative Commons Attribution License (https://creativecommons.org/licenses/by/4.0/), which permits unrestricted use, distribution, and reproduction in any medium, provided the original work, first published in JMIR mHealth and uHealth, is properly cited. The complete bibliographic information, a link to the original publication on https://mhealth.jmir.org/, as well as this copyright and license information must be included. 